\title{
Bogomolny-type equations in curved spacetime
}

\author{
A. Comtet* \\ University of California, Los Angeles, Los Angeles, California 90024 \\ P. Forgács ${ }^{\dagger}$ \\ CERN, Geneva, Switzerland \\ P. A. Horváthy ${ }^{\ddagger}$ \\ Centre de Physique Théorique, Marseille, France \\ (Received 19 December 1983)
}

\begin{abstract}
We show that the field equations of a Yang-Mills-Higgs system in a static curved background metric are solved by the static solutions of first-order equations if the metric satisfies a suitable constraint. Similar results hold also for dyons.
\end{abstract}

In a number of classical field theories there exists a system of first-order equations whose solutions automatically satisfy the second-order equations of motion. For instance, in Yang-Mills-Higgs theories in flat space the minimal-energy static configurations solve the Bogomolny equations $^{1}$

$$
\frac{1}{2} \epsilon_{i j k} F^{j k}=D_{i} \phi \text {. }
$$

All recent developments in monopole theory, ${ }^{2}$ in particular the discovery of explicit solutions with topological charge greater than unity, ${ }^{3}$ are based on (1). In this paper we extend these ideas to gauge theories in a static background metric.

The obvious curved-space generalization of (1) does not work. We were able to modify (1) for metrics satisfying a suitable constraint. This modification, which we call "Bogomolny-type" equations, are closely related to the self-duality equations on a Euclidean manifold, just like in the flat case. ${ }^{4}$ Furthermore, the solutions have minimal energy. This minimal energy is, however, not proportional to the magnetic charge, contrary to what happens in Minkowski space. All the results are easily extended to dyons. ${ }^{5}$ We study finally the problem of compatibility with the Einstein equations in the spherically symmetric case.

Let us consider a static purely magnetic Yang-MillsHiggs (YMH) theory with a compact semisimple gauge group $H$ in a curved background spacetime $\left(M, g_{\mu \nu}\right)$ with the Lagrangian

$-\mathscr{L}=\operatorname{Tr}\left(\frac{1}{4} F_{i j} F^{i j}+\frac{1}{2} D_{i} \phi D^{i} \phi\right) \sqrt{G}$

$$
\left(G=-\operatorname{det} g_{\mu v}\right) .
$$

It is natural to assume that the metric is static, $g_{0 j}=0$, $\partial_{0} g_{j k}=0, \partial_{0} g_{00}=0, j, k=1,2,3$. The field equations read

$$
\begin{aligned}
& \frac{1}{\sqrt{G}} D_{i}\left(\sqrt{G} F^{i j}\right)=\left[\phi, D^{j} \phi\right], \\
& \frac{1}{\sqrt{G}} D_{i}\left(\sqrt{G} D^{i} \phi\right)=0 .
\end{aligned}
$$

We seek a first-order system of equations, which imply (3) and (4). It is not hard to see that the original Bogomolny equations do not work. We try instead a "minimally" modified version of (1):

$$
D_{i} \phi+\left(\partial_{i} u\right) \phi=\sqrt{g} \frac{1}{2} \epsilon_{i j k} F^{j k},
$$

where $u$ is a time-independent function, $g=\operatorname{det}_{i j}$ and $\epsilon_{i j k}=\epsilon^{i j k}$ is the totally antisymmetric (Levi-Civita) symbol. Inserting (5) into (4), using the Bianchi identities $\epsilon^{i j k} D_{i} F_{j k}=0$, the left-hand side (LHS) reads

$$
\begin{aligned}
& {\left[\frac{1}{2} \partial^{i}\left(\ln \left|g_{00}\right|\right)-\partial^{i} u\right] D_{i} \phi} \\
& \left.\quad+\left(\left(\partial^{i} u\right) \partial_{i} \ln \sqrt{\left|g_{00}\right|}-\frac{1}{\sqrt{G}} \partial_{i} \mid \sqrt{G} \partial^{i} u\right)\right) \phi .
\end{aligned}
$$

The coefficient of $D_{i} \phi$ here vanishes if

$$
u=\ln \sqrt{\left|g_{00}\right|} .
$$

The second term becomes

$$
\frac{1}{\sqrt{g}} \partial_{j}\left(\sqrt{g} \partial^{j} u\right) \phi=\left(\Delta \ln \sqrt{\left|g_{00}\right|}\right) \phi .
$$

Equation (4) is hence satisfied if

$$
\Delta \ln \sqrt{\left|g_{00}\right|}=0 \text {. }
$$

As shown by similar calculation, (5)-(7) imply also (3). Thus, we see that any solution of the Bogomolny-type equations

$$
D_{i} \phi+\left(\partial_{i} \ln \sqrt{\left|g_{00}\right|}\right) \phi=\sqrt{g} \frac{1}{2} \epsilon_{i j k} F^{j k}
$$

solves the field equations if the metric satisfies the constraint (7).

The flat-space Bogomolny system corresponds to the static Euclidean self-duality equations (SDE) if $A_{0}$ and $\phi$ are identified. ${ }^{4}$ One may wonder if a similar connection exists also in our case. We show that this is indeed the case.

Let us consider in fact a pure Yang-Mills (YM) theory on the "Euclideanized" manifold $\left(M, g_{\mu \nu}\right)$ (i.e., by changing the sign of $g_{00}$ ) with gauge group $H$ :

(C)1984 The American Physical Society 


$$
\mathscr{L}^{\prime}=\frac{1}{4} \operatorname{Tr} F_{\mu \nu} F^{\mu v} \sqrt{G} .
$$

Equation (9) leads to the field equations

$$
\frac{1}{\sqrt{G}} D_{\mu}\left(\sqrt{G} F^{\mu v}\right)=0 \text {. }
$$

Any solution of the self-duality equations

$$
F_{\mu \nu}= \pm(* F)_{\mu \nu}= \pm \sqrt{G} \frac{1}{2} \epsilon_{\mu \nu \alpha \beta} F^{\alpha \beta}
$$

satisfies, by the Bianchi identities, the field equations (10).

The flat-space version of the aforementioned correspondence breaks down. Define rather

$$
A_{0}=\sqrt{\left|g_{00}\right|} \phi .
$$

Then (9) becomes

$$
\begin{aligned}
& \operatorname{Tr}\left[\left(\frac{1}{4} F_{i j} F^{i j}+\frac{1}{2} D_{i} \phi D^{i} \phi\right) \sqrt{G}+\frac{1}{2} \partial_{j}\left(\phi^{2} \sqrt{g} \partial^{j} \sqrt{\left|g_{00}\right|}\right)\right. \\
& \left.\quad-\frac{\sqrt{G}}{2} \phi^{2} \Delta \ln \sqrt{\left|g_{00}\right|}\right]
\end{aligned}
$$

Equation (13) differs from the YMH Lagrangian (2) by a total divergence if the coefficient of $\phi^{2}$ vanishes.

Hence, we see that the two theories are equivalent if (7) holds. Alternatively, a straightforward calculation shows that the YM equations (10) yield the YMH equations (3) and (4) under the identification (12) exactly when (7) is satisfied. This sheds some light on the reason for our constraint on the metric.

This correspondence provides us with the clue to understand the emergence of the correction term in the Bogomolny-type equation: its LHS is just $\left|g_{00}\right|^{-1 / 2} D_{j}\left(\sqrt{\left|g_{00}\right|} \phi\right)$. Equation (8) is just the selfduality equations (11) expressed in terms of $\phi=\left|g_{00}\right|^{-1 / 2} A_{0}$. The original motivation of Bogomolny in introducing his equations was to prove the stability of monopoles: in flat space the solutions of (1) yield in fact the absolute minima of the energy functional.

For models defined in a general curved background metric the very definition of energy is problematic. For a static space, however, an adequate definition can be given. Indeed, the energy-momentum tensor of a YMH system is

$$
\begin{aligned}
T_{\mu \nu}=\operatorname{Tr} & D_{\mu} \phi D_{\nu} \phi+F_{\mu}^{\rho} F_{\rho \nu} \\
& \left.\quad-\frac{1}{4} g_{\mu \nu} F_{\rho \sigma} F^{\rho \sigma}-\frac{1}{2} g_{\mu \nu} D_{\rho} \phi D^{\rho} \phi\right) .
\end{aligned}
$$

The YMH equations imply that

$$
T_{v ; \mu}^{\mu}=\frac{1}{\sqrt{G}} \partial_{\mu}\left(\sqrt{G} T_{v}^{\mu}\right)-\frac{1}{2} \partial_{\nu} g_{\mu \rho} T^{\mu \rho}=0 .
$$

It follows from (16) and $g_{0 j=0}$ that

$$
T_{0 ; \mu}^{\mu}=\frac{1}{\sqrt{G}} \partial_{\mu}\left(\sqrt{G} T_{0}^{\mu}\right)=0 .
$$

Hence, we get the conserved current $j^{\mu}=\sqrt{G} T_{0}^{\mu}$ whose time component gives, just like in the flat case, the energy

$$
\begin{aligned}
E & =\int d^{3} x \sqrt{G} T_{0}{ }^{0} \\
& =\operatorname{Tr} \int d^{3} x \sqrt{G}\left(\frac{1}{4} F_{i j} F^{i j}+\frac{1}{2} D_{j} \phi D^{j} \phi\right) .
\end{aligned}
$$

From the requirement of finite energy we deduce, for asymptotically flat metrics, the following boundary conditions:

$$
\begin{aligned}
& |\phi|=p_{1}+p_{2} / r+O\left(1 / r^{4}\right), \quad r \rightarrow \infty \\
& \left|D_{j} \phi\right|=0+O\left(1 / r^{2}\right), \quad r \rightarrow \infty .
\end{aligned}
$$

According to (13), the integrand in (17) differs, if (7) is satisfied, from the Yang-Mills Lagrangian $\mathscr{L}^{\prime}(9)$ merely by the total divergence

$$
\frac{1}{2} \operatorname{Tr}_{j}\left(\phi^{2} \sqrt{g} \partial^{j} \sqrt{\left|g_{00}\right|}\right) .
$$

The space integral of (19) is equal to a surface integral on $S_{\infty}^{2}$, where $|\phi|=p_{1}$. Therefore, we have a topological invariant

$$
\begin{aligned}
R & =\frac{1}{2} \operatorname{Tr} \int d^{3} x \partial_{j}\left(\phi^{2} \sqrt{g} \partial^{j} \sqrt{\left|g_{00}\right|}\right) \\
& =\frac{1}{2} p_{1}^{2} \int_{S_{\infty}^{2}} \sqrt{g} \partial^{i} \sqrt{\left|g_{00}\right|} d \sigma_{i} .
\end{aligned}
$$

Equation (20) can also be expressed in terms of the Ricci tensor

$$
R_{0}^{0}=-\frac{1}{\sqrt{\left|g_{00}\right|}} \Delta \sqrt{\left|g_{00}\right|}
$$

in terms of which $R$ reads

$$
R=-\frac{1}{2} p_{1}^{2} \int d^{3} x \sqrt{G} R_{0}^{0} .
$$

It follows from Eq. (7) that $R_{0}^{0}=-\frac{1}{4} \partial_{i}\left(\ln \left|g_{00}\right|\right)$ $\times \partial^{i}\left(\ln \left|g_{00}\right|\right)<0$.

$\mathscr{L}^{\prime}$ is decomposed in turn as

$$
\begin{aligned}
\mathscr{L}^{\prime}= & \frac{\sqrt{G}}{8} \operatorname{Tr}\left\{\left[F_{\mu \nu} \mp(* F)_{\mu \nu}\right]\left[F^{\mu \nu} \mp(* F)^{\mu \nu}\right]\right\} \\
& \pm \frac{\sqrt{G}}{4} \operatorname{Tr} F_{\mu \nu}(* F)^{\mu \nu} .
\end{aligned}
$$

The integral of the second term,

$$
Q^{\prime}=\frac{1}{4} \int d^{3 x} \sqrt{G} \operatorname{Tr} F_{\mu \nu}(* F)^{\mu \nu},
$$

is again invariant under sufficiently smooth deformations $A_{\mu} \rightarrow A_{\mu}+\delta A_{\mu}$.

It follows from the preceding line of thought that the solutions of the Bogomolny-type (i.e., SDE) equations still give the minima of the energy functional: $E_{\min }=Q^{\prime}-R$. Using Eqs. (12) and (18) we get for $Q^{\prime}$

$$
Q^{\prime}=\frac{1}{2} \operatorname{Tr} \int d^{3} x \partial_{j}\left(\sqrt{\left|g_{00}\right|} \epsilon^{j k l} F_{k l} \phi\right)=p_{1} Q,
$$

where

$Q=\operatorname{Tr} \int_{S_{\infty}^{2}} \sqrt{\left|g_{00}\right|} \epsilon^{j k l} F_{k l} \hat{\phi} d \sigma_{j}(\hat{\phi}=\phi /|\phi|)$.

If we use now the Bogomolny-type equations, we can express $Q$ as

$$
Q=\int_{S_{\infty}^{2}} \sqrt{g} \partial^{j}\left(\sqrt{\left|g_{00}\right|}|\phi|\right) d \sigma_{j}
$$

the invariance of $Q$ under deformations is manifest in (25). $Q$ is related to the magnetic charge. To see this we recall 't Hooft's definition of the electromagnetic tensor

$$
\mathscr{F}_{\mu \nu}=\operatorname{Tr}\left\{F_{\mu \nu} \hat{\phi}-(1 / e) \hat{\phi}\left[D_{\mu} \hat{\phi}, D_{\nu} \phi\right]\right\} \text {. }
$$


The magnetic charge is given by

$$
g=\frac{1}{4 \pi} \int_{S_{\infty}^{2}} d \sigma_{i} \frac{1}{2} \epsilon^{i j k} \mathscr{F}_{j k} .
$$

The asymptotic behavior of $D_{\mu} \phi$ implies that

$$
g=\frac{1}{8 \pi} \operatorname{Tr} \int_{S_{\infty}^{2}} \epsilon^{i j k} F_{j k} \hat{\phi} d \sigma_{j} .
$$

For asymptotically flat metrics $\left|g_{00}\right|=1$ on $S_{\infty}^{2}$ so

$$
Q=4 \pi g \text {. }
$$

Notice that the minima of the energy functional are no longer proportional to the magnetic charge unless $R$ vanishes. We get the following formula for $E_{\min }$ :

$$
E_{\min }=-\frac{1}{2} \int d^{3} x \sqrt{g} \Delta\left(\sqrt{\left|g_{00}\right|}|\phi|^{2}\right)+4 \pi g p_{1} .
$$

It is conceivably simpler to solve our first-order system (8) if $\left|g_{00}\right|=1$ when the original Bogomolny form is recovered. Obviously, our metric can always be transformed to such a form by conformal rescaling, $\left.g_{\mu \nu} \rightarrow g_{\mu \nu}^{*}=g_{\mu \nu} / \mid g_{00}\right)$. However, the YMH theory is not conformally invariant in general. The point is that the condition for invariance under rescaling is just the constraint (7). Indeed, the Lagrangian

$$
-\mathscr{L}^{*}=\operatorname{Tr}\left(\frac{1}{4} F_{j k}^{*} F^{* j k}+\frac{1}{2} D_{j}^{*} \phi^{*} D^{* j} \phi^{*}\right) \sqrt{G^{*}}
$$

is transformed to

$$
\begin{aligned}
& \operatorname{Tr}\left(\frac{1}{4} F_{j k} F^{j k}+\frac{1}{2} D_{j} \phi D^{i} \phi\right) \sqrt{G} \\
& \quad+\frac{1}{2} \operatorname{Tr}\left[\partial_{j}\left(\phi^{2} \sqrt{g} \partial^{j} \sqrt{\left|g_{00}\right|}\right)-\phi^{2} \sqrt{G} \Delta \ln \sqrt{\left|g_{00}\right|}\right]
\end{aligned}
$$

if we put

$$
\begin{aligned}
& \phi=\phi^{*} / \sqrt{\left|g_{00}\right|}, \\
& A_{j}=A_{j}^{*} .
\end{aligned}
$$

Plainly, the Bogomolny-type equations (8) are transformed to

$$
D_{i}^{*} \phi^{*}=\sqrt{g^{*}} \epsilon_{i j k} F^{* j k}
$$

under (31).

This is not really surprising if we think in terms of the four-dimensional pure Yang-Mills theory: (30) is manifestly equivalent to a pure YM system $\left(A_{\mu}^{*}, g_{\mu \nu}^{*}\right)$ which in turn is known to be conformally invariant: $\left(A_{\mu}^{*}, g_{\mu \nu}^{*}\right) \sim\left(A_{\mu}, g_{\mu v}\right)$. Finally, we have seen that this latter system is equivalent to the static YMH system $\left(\phi, A_{j}, g_{\mu v}\right)$ if (7) holds.

Our previous results are easily extended to dyons. ${ }^{5}$ In this case $A_{0}$ is different from zero and we make the usual assumption that $\left[A_{0}, \phi\right]=0$.

The field equations now read

$$
\begin{aligned}
& \frac{1}{\sqrt{G}} D_{j}\left(\sqrt{G} F^{j k}\right)=\left[\phi, D^{k} \phi\right]+\left[A_{0}, F^{k 0}\right], \\
& \frac{1}{\sqrt{G}} D_{j}\left(\sqrt{G} F^{j 0}\right)=0 \\
& \frac{1}{\sqrt{G}} D_{j}\left(\sqrt{G} D^{j} \phi\right)=0 .
\end{aligned}
$$

It has been observed that in flat space one can look for a solution of (32a)-(32c) with the ansatz $A_{0}=c \phi$ (where $C=$ const) when (32b) and (32c) become proportional. The same thing happens with the modified ansatz

$$
A_{0}=C \sqrt{\left|g_{00}\right|} \phi
$$

if the constraint (7) is satisfied. This can be verified by a straightforward calculation analogous to the one we did before in the purely magnetic case.

Equation (32a) becomes then

$$
\frac{1}{\sqrt{G}} D_{j}\left(\sqrt{G} F^{j k}\right)=\left(1-C^{2}\right)\left[\phi, D^{k} \phi\right]
$$

and the field equations $(32 a)-(32 c)$ are solved by the Bogomolny-type equations:

$\sqrt{g} \frac{1}{2} \epsilon_{i j k} F^{j k}=\sqrt{1-C^{2}}\left[D_{i} \phi+\partial_{i}\left(\ln \sqrt{\left|g_{00}\right|}\right) \phi\right]$,

$F_{0 i}=C \sqrt{\left|g_{00}\right|}\left[D_{i} \phi+\partial_{i}\left(\ln \sqrt{\left|g_{00}\right|}\right) \phi\right]$.

The electric charge of the dyon is given by

$q=\operatorname{Tr} \int d^{3} x \partial_{i}\left(\sqrt{g} F_{0}^{i} \hat{\phi}\right)=\operatorname{Tr} \int_{S_{\infty}^{2}} d \sigma_{i}\left(\sqrt{G} F^{i 0} \hat{\phi}\right)$,

where we assumed again that

$$
\left|g_{00}\right|=1 \text { on } S_{\infty}^{2} \text {. }
$$

The energy is expressed now as

$E=\operatorname{Tr} \int d^{3} x \sqrt{G}\left(\frac{1}{4} F_{i j} F^{i j}-\frac{1}{2} F_{0 j} F^{0 j}+\frac{1}{2} D_{i} \phi D^{i} \phi\right)$.

Decomposing (37) analogously to (23) we conclude that solutions of the first-order system (34) and (35) give the absolute minima of the energy in a fixed magnetic and electric charge sector. These minima are

$$
E_{\min }=4 \pi p_{1}\left(q^{2}+g^{2}\right)^{1 / 2}+\frac{1}{2} p_{1}^{2} \int d^{3} x \sqrt{G} R_{0}^{0} .
$$

In our paper, the constraint (7) plays a crucial role. It is beyond the scope of the present paper to classify the solutions of (7).

In the case of spherical symmetry this problem can be solved easily. Indeed let us consider

$$
g_{\mu \nu}=\operatorname{diag}\left(-e^{v}, e^{\lambda}, r^{2}, r^{2} \sin ^{2} \theta\right) .
$$

The constraint (7) now reads

$$
\frac{1}{2} v^{\prime \prime}+\frac{1}{r} v^{\prime}-\frac{1}{4} v^{\prime} \lambda^{\prime}=0 \text {. }
$$

Equation (39) can be integrated easily. Making the usual spherically symmetric ansatz for $A_{\mu}$

$$
A_{0}=0, \quad A_{i}^{a}=\epsilon_{i a b} \frac{x^{b}}{r^{2}}[v(r)-1], \quad \phi^{a}=\frac{x^{a}}{r} p(r)
$$

our first-order system (5) becomes

$$
\begin{aligned}
& v^{\prime}=v p e^{\lambda / 2}, \\
& p^{\prime}=\frac{v^{2}-1}{r^{2}} e^{\lambda / 2}-p v^{\prime} / 2 .
\end{aligned}
$$

Equation (39), (40a) and (40b) were first studied in Ref. 6. Up to now we limited ourselves to the curved-space YMH equations in a prescribed background metric. Our results 
simplify a great deal the problem of finding explicit solutions in the static case without further restriction, like spherical symmetry. ${ }^{7}$

As a next step it is natural to ask if our procedure can be applied to the coupled Einstein-Yang-Mills-Higgs equations. This depends only on the compatibility of the constraint (7) with the Einstein equations. We have no complete answer to this question as yet. In the spherically symmetric case it turns out that the only allowed solution is the one studied in Ref. 8. The details of our calculations are given in the appendix.

\section{ACKNOWLEDGMENTS}

Two of us (P. F. and P. A. H.) are indebted to J. M. Souriau for hospitality in Marseille where part of this work has been completed. Discussions are acknowledged with A. Chakrabarti and J. L. Richard.

\section{APPENDIX}

The coupled Einstein-Yang-Mills-Higgs equations read ${ }^{8}$

$$
\begin{aligned}
& y^{\prime}=2 r U_{1}, \\
& {\left[r\left(e^{y}-e^{x}\right)\right]^{\prime}=2 r^{2} e^{y}\left(U_{1}+U_{2}\right),} \\
& \left(v^{\prime} e^{x}\right)^{\prime}=e^{y}\left[\frac{v\left(v^{2}-1\right)}{r^{2}}+v p^{2}\right], \\
& \left(r^{2} p^{\prime} e^{x}\right)^{\prime}=e^{y} 2 v^{2} p,
\end{aligned}
$$

where

$$
\begin{aligned}
& U_{1}=\frac{\left(v^{\prime}\right)^{2}}{r^{2}}+\frac{\left(p^{\prime}\right)^{2}}{2}, \quad U_{2}=\frac{\left(v^{2}-1\right)^{2}}{2 r^{4}}+\frac{v^{2} p^{2}}{r^{2}}, \\
& y=\frac{1}{2}(v+\lambda), \quad x=\frac{1}{2}(v-\lambda) .
\end{aligned}
$$

(The gauge and gravitational coupling constants have been taken equal to 1 .)

We study now the compatibility of Eqs. (41a) and (41b) with (39), (40a) and (40b). It is a straightforward although somewhat tedious procedure. Our task is greatly simplified if we use the integrated version of (39),

$$
(x+y)^{\prime}=\frac{a}{r^{2}} e^{(y-x) / 2} .
$$

The finite-energy boundary conditions $v \rightarrow 0$ and $p \rightarrow 1$ as $r \rightarrow \infty$ fix the value of the constant of integration to be $a=-2$.

Adding (41a) and (41b) and using (43) we end up with the following integrability condition:

$$
Z^{2}\left[p^{2}+2 p\left(v^{2}-1\right)+r^{2}\right]+2 r Z-r^{2}=0,
$$

where we introduced $Z=e^{(y-x) / 2}$.

Differentiating (44) we get yet another integrability condition, which is again an algebraic equation in

$$
\begin{aligned}
Z^{4}\left[\left[p^{2}+2 p\left(v^{2}-1\right)+r^{2}\right]\right. & {\left.\left[\frac{4 v^{2} p^{2}}{r}+2 \frac{\left(v^{2}-1\right)^{2}}{r^{3}}+\frac{p^{2}}{r^{3}}+\frac{2 p}{r^{3}}\left(v^{2}-1\right)\right]-\frac{1}{r}\right] } \\
& +Z^{3}\left[8 v^{2} p^{2}-1+\frac{4\left(v^{2}-1\right)^{2}}{r^{2}}+\frac{3 p^{2}}{r^{2}}+6 p \frac{\left(v^{2}-1\right)}{r^{2}}\right]+Z^{2}\left[\frac{p^{2}}{r}+2 p \frac{\left(v^{2}-1\right)}{r}+3 r\right]+3 Z-2 r=0 .
\end{aligned}
$$

Equations (44) and (45) are compatible only if $v=0$ and $p=1$. The physically admissible (positive) solution of (44) yields in fact the extreme Nordström-Reissner metric ${ }^{10}$

$$
e^{v}=e^{-\lambda}=\left(1-\frac{1}{r}\right)^{2} \text {. }
$$

${ }^{*}$ Permanent address: Division de Physique Théorique, Institut de Physique Nucléaire, Orsay, France.

${ }^{\dagger}$ Permanent address: Central Research Institut for Physics, Budapest, Hungary.

†Permanent address: Centre de Physique Théorique, CNRS Luminy Case 907, F-13288 Marseille, Cedex 9 France.

${ }^{1}$ E. B. Bogomolny, Yad. Fiz. 24, 861 (1976) [Sov. J. Nucl. Phys. 24, 449 (1976)]; S. Coleman, S. Park, A. Neveu, and C. M. Sommerfield, Phys. Rev. D 12, 544 (1977); for a review see, e.g., D. Maison, talk delivered at the 1983 Karpacz Winter School (unpublished).

${ }^{2}$ G. 't Hooft, Nucl. Phys. B79, 276 (1974); A. M. Polyakov, Pis'ma Zh. Eksp. Teor. Fiz. 20, 430 (1974) [JETP Lett. 20, 194 (1974)].

${ }^{3}$ P. Forgács, Z. Horváth, and L. Palla, Phys. Lett. 99B, 232
(1981); R. Ward, Commun. Math. Phys. 79, 317 (1981); see also Monopoles in Quantum Field Theory, proceedings of the Monopole Meeting Italy, Trieste 1981, edited by N. S. Craigie, P. Goddard, and W. Nahm (World Scientific, Singapore, 1982).

${ }^{4}$ N. Manton, Nucl. Phys. B135, 319 (1978); P. Forgács, and N. Manton, Commun. Math. Phys. 72, 15 (1980).

${ }^{5}$ B. Julia and A. Zee, Phys. Rev. D 11, 2227 (1975).

${ }^{6}$ A. Comtet, Ann. Inst. Henri Poincaré 32, 283 (1980).

${ }^{7}$ E. J. Weinberg and A. H. Guth, Phys. Rev. D 14, 1660 (1976).

${ }^{8}$ F. A. Bais and R. J. Russel, Phys. Rev. D 11, 2692 (1975); Y. M. Cho and P. G. O. Freund, ibid. 12, 1588 (1975).

${ }^{9}$ P. van Nieuwenhuizen, D. Wilkinson, and M. J. Perry, Phys. Rev. D 13, 778 (1976).

${ }^{10}$ G. W. Gibbons and C. M. Hull, Phys. Lett. 109B, 190 (1982). 